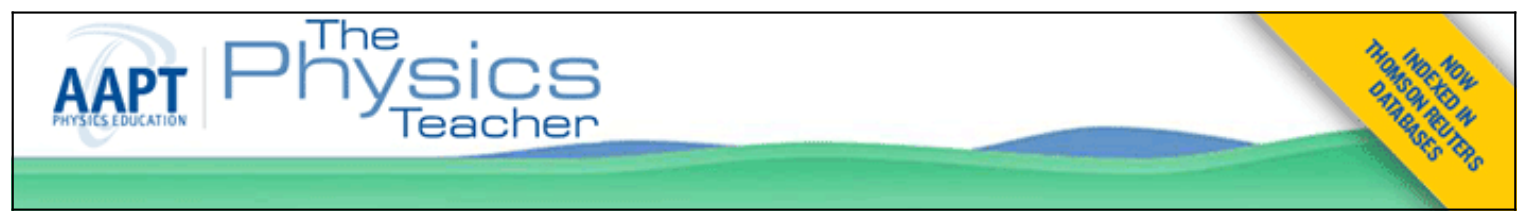

\title{
Pressure Oscillations in Adiabatic Compression
}

Roland Stout

Citation: The Physics Teacher 49, 280 (2011); doi: 10.1119/1.3578419

View online: http://dx.doi.org/10.1119/1.3578419

View Table of Contents: http://scitation.aip.org/content/aapt/journal/tpt/49/5?ver=pdfcov

Published by the American Association of Physics Teachers

\section{Articles you may be interested in}

Apparatus for demonstrating longitudinal wave pulses

Phys. Teach. 49, 312 (2011); 10.1119/1.3578431

Apparatus Named After Our Academic Ancestors - II

Phys. Teach. 49, 28 (2011); 10.1119/1.3527751

Illustrating Electric Circuit Concepts with the Glitter Circuit

Phys. Teach. 42, 359 (2004); 10.1119/1.1790344

A low-cost audio oscillator

Phys. Teach. 16, 303 (1978); 10.1119/1.2339949

A pie plate radio

Phys. Teach. 16, 302 (1978); 10.1119/1.2339948

\section{Collect Clean, Repeatable,} and Noise-Resistant

\section{Motion Data}

Vernier Dynamics Cart and Track System with Motion Encoder

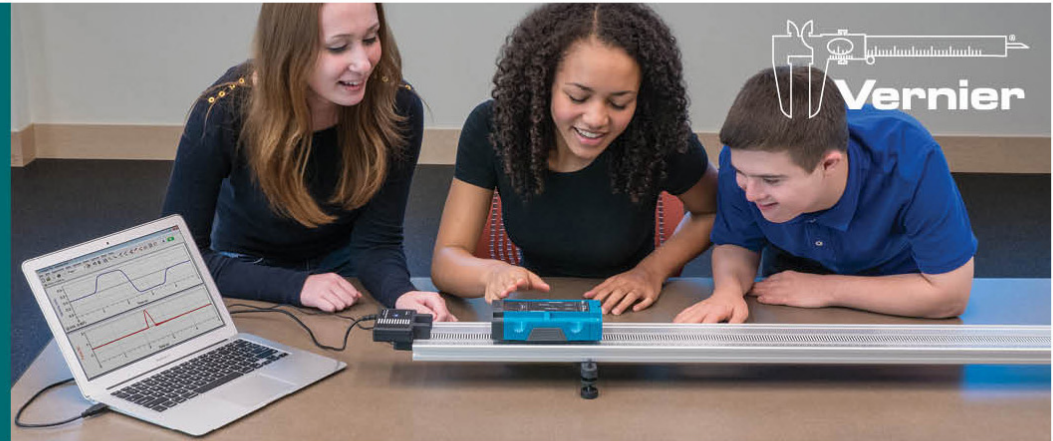




\section{Pressure Oscillations in Adiabatic Compression}

Roland Stout, University of North Carolina Pembroke, Pembroke, NC

A fter finding Moloney and McGarvey's ${ }^{1}$ modified adiabatic compression apparatus, I decided to insert this experiment into my physical chemistry laboratory at the last minute, replacing a problematic experiment. With insufficient time to build the apparatus, we placed a bottle between two thick textbooks and compressed it with a third textbook forced down from above. We discovered that approximately one in three trials exhibited a damped pressure oscillation after compression, as shown in Fig. 1 below, making it difficult to determine the true peak pressure. We discarded these trials and used only those without oscillation. I subsequently have had time to build a version of Moloney and McGarvin's apparatus. Using this apparatus, my students and I have recently performed numerous adiabatic compression trials with three different gases, with very few instances of oscillation.

Figure 1 indicates that the frequency of oscillation is around $20 \mathrm{~Hz}$ for a 2-L bottle. ${ }^{2}$ This is well below the fundamental frequency of the bottle, which was found to be $96 \mathrm{~Hz}$. We discovered that when compressed, mechanical driving on the side of the plastic bottle induced a resonance at $18.5 \mathrm{~Hz}$. I suspect that this is an oscillation in the plastic bottle but have done no other

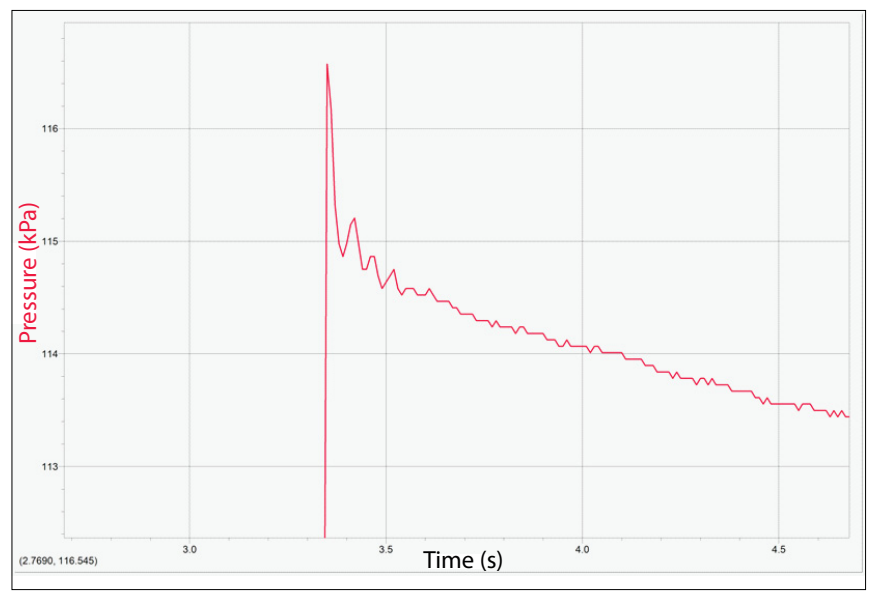

Fig. 1. Expansion of the region of the maximum pressure reached during a symmetric, adiabatic compression showing a damped oscillation in the pressure.

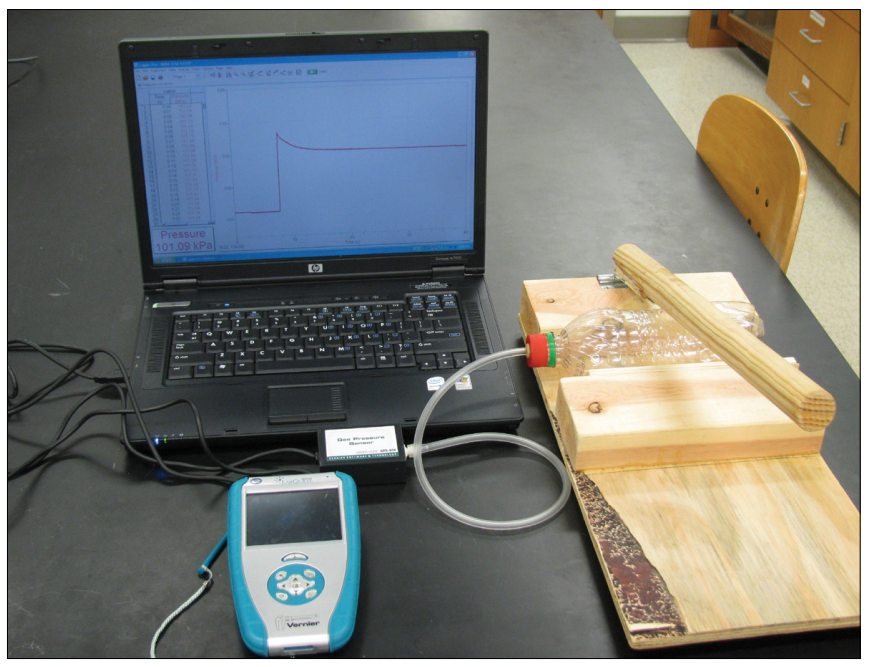

Fig. 2 Adiabatic Compression apparatus for a 20-oz bottle size. Shown are the homemade apparatus, the pressure sensor and LabQuest interface from Vernier Software \& Technology, and a computer running Vernier's Logger Pro program showing the pressure trace for an adiabatic compression trial.

work seeking to verify this.

Though these pressure oscillations are unhelpful in the adiabatic compression experiment, they also offer an opportunity. They lead to an interesting discussion of curve fitting.

\section{References}

1. M. J. Moloney and A. P. McGarvey, "A simplified adiabatic compression apparatus," Phys. Teach. 45, 452-453 (Oct. 2007).

2. Some students had insufficient strength to compress a 2-L bottle. We now use primarily $20-\mathrm{oz}$ bottles.

Roland Stout, associate professor of chemistry, stands on the false boundary between chemistry and physics. Though formally a physical chemist, he was trained as a chemical physicist and is equally conversant with physicists and chemists.

Department of Chemistry and Physics, University of North Carolina at Pembroke, Pembroke, NC 28372-1510; roland.stout@uncp.edu

A supplemental simulation for the article by Roland Stout is The Hard Disk PVT System in the OSP ComPADRE Collection, which simulates a two-dimensional system of particles confined between a thermal reservoir and a piston. This computer model is designed to study the equation of state for hard disks. Slow-moving particles are color-coded as blue, and fast particles are color-coded as yellow. Users can set the initial particle kinetic energy, the initial particle separation, and the thermal reservoir temperature. The thermal conductivity parameter $\kappa$ determines the probability that a collision with the thermal wall will affect (thermalize) the colliding particle. If the conductivity is one, the particle velocity after a reservoir collision is set according to Maxwell-Boltzmann statistics and the system will equilibrate with the reservoir. The internal energy of the system will, however, fluctuate. If the conductivity is zero, all reservoir collisions are elastic and the internal energy is conserved. (continued on next page) 
Particles in this model have unit mass and interact through contact forces. Boltzmann's constant is also unity. Collision times are computed analytically because particles and pistons move with constant velocity between collisions. The time evolution algorithm advances the particle and piston positions from collision to collision until the requested time step $\Delta t$ is achieved. The time evolution is then paused, data are accumulated, and the screen is redrawn.

The piston input fields are used to compress and expand the system. Users can approximate an ideal gas by set-

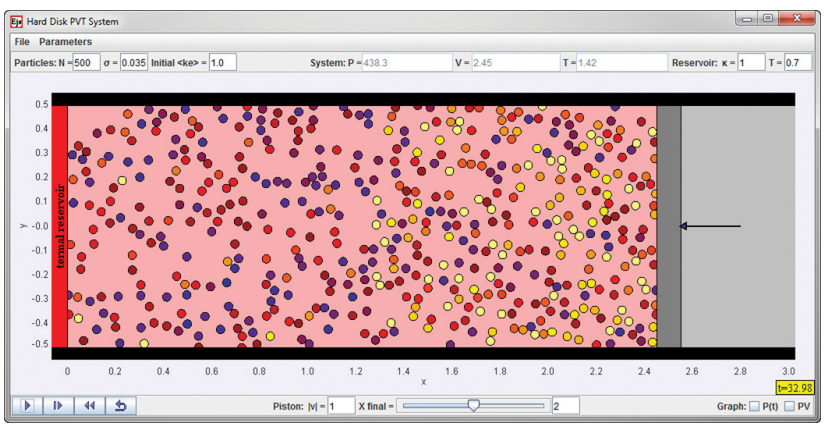
ting the particle diameter $\sigma$ to a small value. Users can approximate an adiabatic compression by setting the thermal conductivity $\kappa=0$ and can approximate an isothermal compression by setting $\kappa=1$. The piston speed $|v|$ must, of course, be small to insure that the system remains in equilibrium during the compression (quasi-static approximation) for a reversible process. When the piston speed is large (see screenshot), nonuniform pressure and density are clearly observed. Separate windows allow users to follow the change in state of the system on a PV plot or a time evolution plot.

www.compadre.org/OSP/items/detail.cfm?ID=10855

A Lennard-Jones version of the PVT model is also available. www.compadre.org/OSP/items/detail.cfm?ID=10857

Because the numerical solution of coupled differential equations is more difficult than the hard disk collision time computation, fewer particles can be simulated in the Lennard-Jones model. Related items for these models can be found in the OSP Collection by searching for "molecular dynamics" or "kinetic theory."

These supplemental simulations for the paper by Roland Stout have been approved by the author and the TPT editor. Partial funding for the development of this model was obtained through NSF grant DUE-0937731.

-Wolfgang Christian 